\title{
Reliability of environmental sampling to quantify Mycobacterium avium subspecies paratuberculosis on California free-stall dairies
}

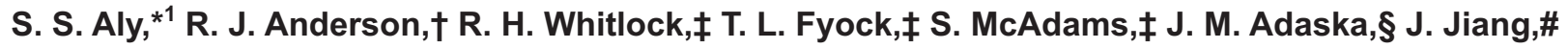 \\ and I. A. Gardner* \\ ${ }^{*}$ Department of Medicine and Epidemiology, School of Veterinary Medicine, University of California, Davis 95616 \\ †California Department of Food and Agriculture, Animal Health Branch, Sacramento 95814 \\ łJohne’s Research Laboratory, New Bolton Center, School of Veterinary Medicine, University of Pennsylvania, 382 West Street Road, \\ Kennett Square 19348 \\ §California Animal Health and Food Safety Laboratory, Tulare Branch, 18830 Road 112, Tulare 93274 \\ \#Department of Statistics, University of California, Davis 95616
}

\begin{abstract}
The reliability of environmental sampling to quantify Mycobacterium avium ssp. paratuberculosis (MAP) based on collector and time was evaluated. Fecal slurry samples were collected using a standardized protocol simultaneously by 2 collectors of different experience levels. Samples were collected from 30 cow pens on 4 dairies every other day on 3 occasions while cow movements between pens were minimal. The 4 study herds had moderate MAP seroprevalence and were housed in free-stall dairies in central California. Results of testing the environmental samples for MAP using PCR and culture were strongly correlated. The reliability of environmental sampling simultaneously by different collectors as estimated by the intraclass correlation coefficient was excellent (81\%) for PCR and good (67\%) for culture and may justify comparison of quantitative results of samples collected by different investigators. The reliability of environmental sampling over a 5 -d period was good (67 and $64 \%$ for PCR and culture results, respectively), which justifies the utility of environmental sampling to identify pens with a high MAP bioburden between routine cow pen changes on a dairy. Environmental sampling of free-stall pens using the standardized sampling protocol yielded comparable PCR and culture results across collectors with different experience levels and at different times within a 5 -d period.
\end{abstract}

Key words: reliability, environmental sampling, $M y$ cobacterium avium ssp. paratuberculosis, quantitative real-time PCR

\section{INTRODUCTION}

Environmental samples, such as fecal slurry from dairy pens, can be used to detect Mycobacterium avium

Received September 2, 2008.

Accepted March 22, 2009.

${ }^{1}$ Corresponding author: saly@ucdavis.edu ssp. paratuberculosis (MAP). In the United States, environmental samples are used for classification of MAP herd status for the Voluntary Bovine Johne's Disease Control Program. The Voluntary Bovine Johne's Disease Control Program allows MAP-negative environmental samples as an alternative to MAP antibodynegative samples from 30 individual cows for entry into the test-negative component of the program (USDA, 2006). Environmental samples were also used in the National Animal Health and Monitoring System Dairy 2002 study (USDA, 2005) and Dairy 2007 study (USDA, 2008a) to estimate the national herd-level prevalence. The sensitivity of the nonstandardized environmental sampling protocol used in the Dairy 2002 study to detect herds with at least 1 serum ELISA-positive result was estimated to be $76 \%$ (Lombard et al., 2006).

Recently, a standardized sampling protocol to detect MAP on a dairy using a single investigator collecting environmental samples from common locations was found to have comparable herd sensitivity to ELISA testing and pooled fecal culture (Berghaus et al., 2006). In addition, findings from a simulation study comparing 5 testing strategies indicated that MAP culture of 6 to 10 environmental samples was the most costeffective method for initial classification of MAP herd status in moderate- and high-prevalence herds and of comparable cost and sensitivity with ELISA in lowprevalence herds in the Midwest of the United States (Tavornpanich et al., 2008).

The use of quantitative tests to estimate MAP concentrations in environmental samples collected from individual pens, rather than common locations such as the wastewater lagoon and return alleyway, may be used to rank pens on a dairy according to the MAP bioburden. Subsequently, cows in pens with the highest environmental MAP bioburden may be preferentially tested, provided MAP concentrations in pen environmental samples can be estimated before routine moving of cows. Typically, cows are routinely moved between 
pens on a California dairy every 1 to 2 wk to adjust for changes in milk production and feed rations; hence, moving may result in a substantial change in pen environmental MAP concentrations. If quantitative realtime PCR (qrt-PCR) results could be correlated with culture results on Herrold's egg yolk medium (HEYM), which is often considered the most appropriate reference test for MAP in live animals (Collins et al., 2006), then qrt-PCR might be used as a rapid test to rank pens by MAP bioburden.

The utility of environmental samples beyond classifying a herd as MAP infected or not depends in part on the reliability of sample collection and processing, both of which can be characterized in a sampling protocol. A reliable environmental sampling protocol yields samples with less variability attributable to the collection procedure, including collector and time of sampling, than variability attributable to true differences between samples; hence, it yields samples that are comparable and representative of MAP fecal shedding by the cow populations sampled. Other sources of variability in environmental samples are related to cow factors and laboratory testing.

The use of a standardized sampling protocol (Berghaus et al., 2006) should minimize variability through implementation of a specific sequenced procedure that increases the consistency and uniformity with which samples are collected, processed, handled, and shipped. However, the reliability of environmental sampling when performed by different collectors, such as from several herds in a region or from the same herd over time, has not been studied to our knowledge. In addition, the ideal time for collection of environmental samples representative of the current pen population, and changes in MAP concentration over time in a pen, may be investigated through testing environmental samples collected repeatedly over time and when movement of cows between pens is absent or minimal.

The objective of this longitudinal study was to estimate the reliability of environmental sampling to quantify MAP concentrations in fecal slurry samples from 4 free-stall California dairies based on collector and time while adjusting for pen and dairy sampled. Samples were collected using a standardized sampling protocol and were evaluated by qrt-PCR and culture on HEYM.

\section{MATERIALS AND METHODS}

\section{Study Herds}

Four central California dairies were enrolled in the study. Both dairy 1 (1,676 Holsteins) and dairy 2 (3,577 Jerseys) had routine fecal cultures and serum
ELISA testing for MAP at dry-off. Dairies 1 and 2 had a moderate seroprevalence of MAP of 3.5 and $4.5 \%$, respectively, based on ELISA testing at dry-off. In addition, environmental samples were collected and cultured quarterly for MAP as part of the National Johne's Disease Demonstration Herd Project (USDA, 2002). More than $80 \%$ of fecal slurry samples collected from these herds in 2006 and 2007 were MAP positive on culture. Dairies 3 and 4 (1,326 and 1,166 Holsteins, respectively) were candidate herds from earlier Johne's disease studies and had whole-herd ELISA testing performed in 2004 and 2006, with similar MAP seroprevalence of 3.8 and $4.6 \%$, respectively, based on the whole-herd ELISA tests.

Lactating cows on all 4 dairies were housed in freestall pens that were flushed with wastewater from the storage lagoon. Cows on dairy 1 were moved between pens once every 2 wk based on changes in milk production, whereas cows were moved out of the fresh-cow pen every 1 to 2 wk, depending on pen density. On dairy 2, cows were moved at the end of each week, and in dairies 3 and 4 cows were moved at the beginning of the week. On each dairy, environmental samples were collected for the purpose of this study from all the pens housing the entire adult cow herd, specifically from 8, 11, 7, and 4 pens from dairies $1,2,3$, and 4 , respectively. Cow numbers ranged from 105 to 418 cows per pen, with a median of 226, 255, 195, and 301 cows on dairies 1, 2, 3 , and 4 , respectively.

\section{Study Period}

Environmental samples were collected every other day on 3 different occasions from dairies 1 and 2 between November 16 and November 21, 2006, and from dairies 3 and 4 between May 30 and June 3, 2007. Sampling dates were selected to be immediately after routine moving of cows between pens. Herd managers were also asked to minimize moving cows between pens sampled during the study period, with the exception of new hospital entries and discharges, which the managers were requested to document and report to collectors.

\section{Collection of Environmental Samples}

Sample collection began 1 to $2 \mathrm{~d}$ after scraping the concrete pathways connecting the 2 sides of each free-stall pen (crossover alleyways) to avoid sampling fecal slurry unrepresentative of cows housed in the sampled pens and took place in the mornings at approximately the same time. Two veterinarians (RA and SA) simultaneously collected environmental samples from all 4 dairies. Collector 1 (RA) regularly collected environmental samples quarterly from California herds 
enrolled in the National Johne's Disease Demonstration Herd Project using the standardized sampling protocol described by Berghaus et al. (2006). Collector 2 (SA) had not collected any environmental samples before this study and had received 1 training session by RA after reviewing the same standardized sampling protocol, approximately 1 mo before commencement of the study. During the training session, RA demonstrated sampling the lagoon and scraping the surface of a crossover alley. The lagoon was sampled from only 2 of the study dairies because of safety concerns; hence, all lagoon results were excluded from the analysis.

On the first day of sampling (d 1) in all study herds, and with the order maintained throughout the remaining sampling days (d 3 and 5), cow pens were numbered from 1 to $n$, beginning with the lactating pens, followed by dry pens and finally the hospital string. Samples were labeled by herd, date of collection, cow pen, and collector (coded to maintain blinding at the laboratory). Collectors wore disposable plastic overboots between pens to avoid carryover contamination from previously sampled pens.

Approximately $10 \mathrm{~mL}$ of fecal slurry was collected by hand approximately every $1 \mathrm{~m}$ along the crossover alleyways where the flush system had not removed slurry and around water troughs. Individual cow fecal pats were deliberately avoided. Each collector began at a different end of the free-stall pen and spent a comparable time sampling crossover alleyways until the entire surface had been systematically sampled. Environmental samples were placed in a 1.9-L plastic container that was cleaned, disinfected with a quaternary ammonium compound, thoroughly rinsed, and dried with disposable paper towels after each pen. After the final collection from any pen, the slurry was mixed with a sterile wooden tongue depressor by 10 vertical stirs from the bottom to the top, followed by 10 clockwise and 10 anticlockwise stirs. A 40-mL sample was transferred into sterile polypropylene conical tubes individually wrapped in polyethylene sterile bags (Whirl-Pak, Nasco, Ft. Atkinson, WI), 1 tube for HEYM culture and MAP qrt-PCR testing and 2 tubes stored as repository split samples at $-70^{\circ} \mathrm{C}$. Samples were packaged with ice packs and shipped by overnight delivery to the Johne's Research Laboratory at the University of Pennsylvania, which is approved for MAP culture and qrt-PCR by USDA-National Veterinary Services Laboratories (USDA, 2008b).

\section{Laboratory Testing}

Environmental samples collected by RA and SA on a given day were processed and tested in parallel. Environmental samples were split into 2 aliquots at the laboratory: 1 aliquot was tested using VetAlert, a qrtPCR kit (Tetracore Inc., Rockville, MD) that targets the single-copy hspX gene, and cultured on HEYM. The second aliquot was frozen at $-70^{\circ} \mathrm{C}$ for future testing if needed. For each environmental sample, $2 \mathrm{~g}$ was placed into a $50-\mathrm{mL}$ conical tube containing $35 \mathrm{~mL}$ of sterile water. Each sample was rocked for $30 \mathrm{~min}$ and then allowed to settle for $30 \mathrm{~min}$. Twenty-five milliliters was then removed from the tube, $20 \mathrm{~mL}$ of which was placed in a new sterile $50-\mathrm{mL}$ conical tube for qrt-PCR, and the remaining $5 \mathrm{~mL}$ of which was used for culture.

Qrt-PCR. The qrt-PCR samples were then centrifuged at $2,500 \times g$ for $10 \mathrm{~min}$ at $24^{\circ} \mathrm{C}$. Eighteen milliliters was decanted from the tube and discarded. Each sample was then vortexed to resuspend the pellet in the remaining $2 \mathrm{~mL}$ of fluid and was transferred to a bead-beater tube. The samples were beat for $5 \mathrm{~min}$ to lyse the cells. The DNA was extracted according to the directions of the manufacturer (Tetracore, 2008). The MAP qrt-PCR was performed on each DNA sample by using a SmartCycler (Cepheid, Sunnyvale, CA) according to the directions of the manufacturer. Each qrtPCR run consisted of 6 environmental samples tested in duplicate, a 25,000 genomic copy standard provided in the kit, an internal positive control sample from the extraction, and 2 negative control wells consisting of $2.5 \mu \mathrm{L}$ each of $1 \times \mathrm{TE}(10 \mathrm{~m} M$ Tris-hydrochloric, $\mathrm{pH}$ 8.0; $1 \mathrm{~m} M$ EDTA), for a total of 16 wells. Results were reported as cycles-to-threshold $(\mathbf{C t})$ with a maximum value of 50. On the basis of analysis of a standard curve of serial dilutions of genomic MAP DNA, a 1-log dilution was equivalent to approximately a 3.3 higher $\mathrm{Ct}$ value. The mean $\mathrm{Ct}$ value for the duplicates was used for data analysis.

Bacterial Culture. Each sample was cultured on 4 HEYM tubes, each containing mycobactin, using a centrifugation culture procedure, as described previously and as routinely used in the laboratory (Crossley et al., 2005). Three known positive controls were processed and cultured with environmental samples from each day. The number of colony-forming units in each tube was counted by a different technician every 2 wk for 16 wk and was based on noncontaminated tubes. The natural $\log (\mathbf{l n})$ transformation of the mean colonyforming units after addition of 0.5 was used for data analysis.

\section{Statistical Analysis}

Descriptive Statistics. Descriptive statistics were generated for the qrt-PCR and ln HEYM culture results for MAP in environmental samples. The correlation of mean Ct values with mean colony-forming units per tube and ln mean colony-forming units per tube was 


\section{$\begin{array}{llll}\text { Dairy } 1 & \text { Dairy } 2 & \text { Dairy } 3 & \text { Dairy } 4\end{array}$}

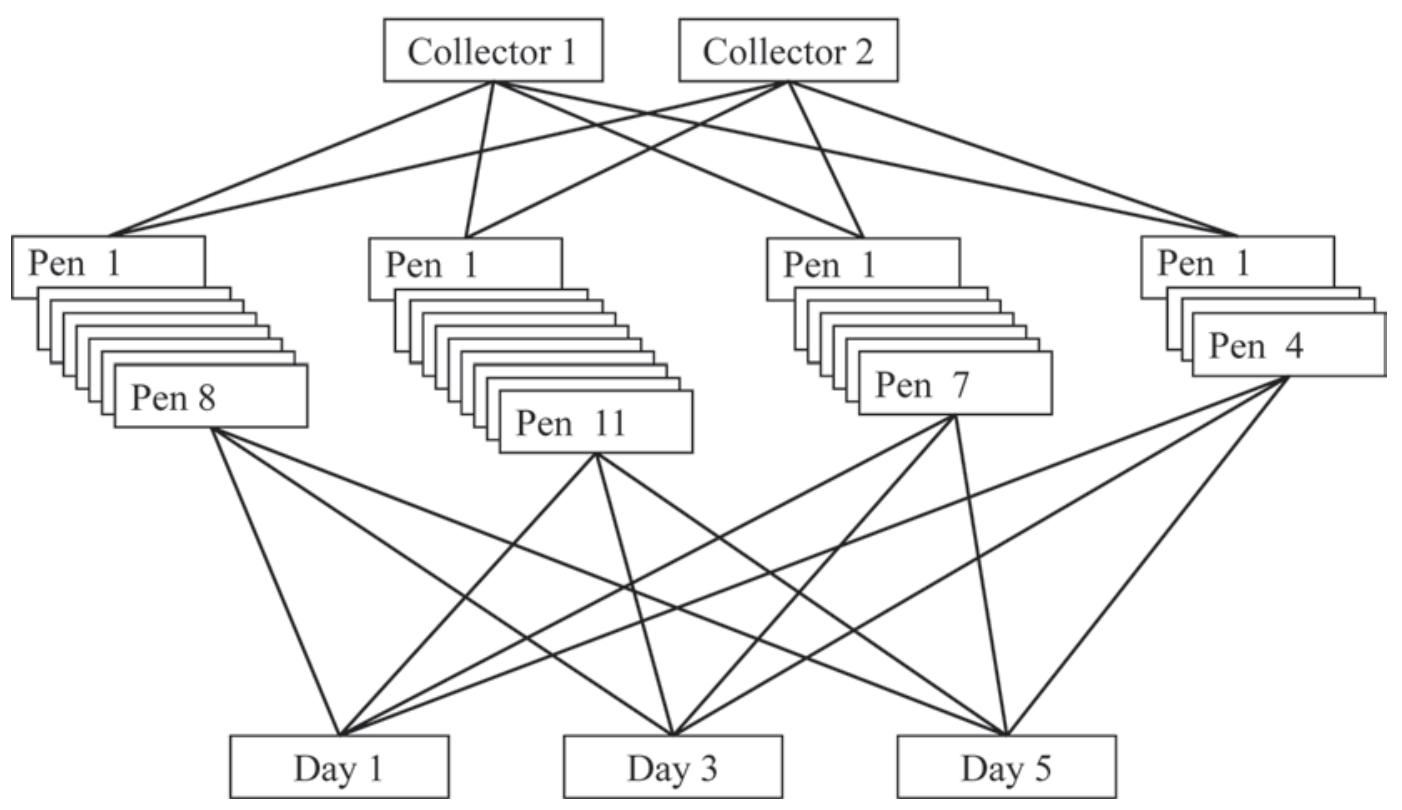

Figure 1. Schematic presentation of a longitudinal study of the reliability of environmental sampling and testing methods to quantify Mycobacterium avium ssp. paratuberculosis in cow pens in 4 California free-stall dairies. Pens were nested within dairy and crossed with collector and day of sampling.

estimated using the Spearman's rank correlation coefficient and Pearson correlation coefficient, respectively. Histograms and normal quantile plots were used to assess the normality of the mean $\mathrm{Ct}$ values and $\mathrm{ln}$ mean colony-forming units per tube. The differences between collectors (RA subtracted from SA) for each of qrt-PCR and the ln mean HEYM culture results were plotted against their respective means and were compared with the mean of all differences and the $95 \%$ limits of agreement in the Bland and Altman plots. The $95 \%$ limits of agreement were calculated as the mean difference \pm 1.96 SD of the differences (Bland and Altman, 1986).

Variance Components Models. For each outcome, there were 4 factor levels: dairy, pen, collector, and day (Figure 1). Pens were nested within dairy. In dairy $i, i$ $=\{1,2,3,4\}$ were pens $j$, where for $i=1, j=\{1, . ., 8\}$, for $i=2, j=\{1, . ., 11\}$, for $i=3, j=\{1, . ., 7\}$, and for $i=4$, $j=\{1, \ldots, 4\}$. Pens were cross-classified by collector $k ; k$ $=\{1,2\}$; and day $l ; l=\{1,2,3\}$.

Variance components models with random intercepts for dairy $u_{i}$ and pen $v_{i j}$ were used to account for variability attributable to dairies and pens within dairies, respectively. The cross-classification of pens by collector and day was addressed by including random intercepts, $w_{k}$ for collector and $z_{l}$ for day (Goldstein, 1987; Marchenko, 2006). All random effects $\left(u_{i}, v_{i j}, w_{k}, z_{l}\right)$ and residual error $\left(e_{i j k l}\right)$ were assumed to be normally distributed with mean $=0$ and variances $\sigma_{u}^{2}, \sigma_{v}^{2}, \sigma_{w}^{2}, \sigma_{z}^{2}$, and $\sigma^{2}$, respectively. For each study outcome $\left(y_{i j k l}\right)$, Ct values or ln mean colony-forming units per tube, variance components were estimated from a multilevel mixed model (equation [1]):

$$
y_{i j k l}=\beta_{0}+u_{i}+v_{i j}+w_{k}+z_{l}+e_{i j k l} .
$$

Models were fit using REML (Jiang, 2007). Quantile plots of model residuals and standardized residuals were evaluated for normality. Similarly, the empirical BLUP for dairy, pen, collector, and day from both qrtPCR and HEYM models were estimated and evaluated for normality.

Estimates of variability attributable to collector, day, dairy, and pen as a percentage of the total variability were computed for each test result. Reliability was assessed using the intraclass correlation coefficient (ICC), an estimate of similarity in results of samples from the same group. The following 3 ICC were estimated:

$$
\text { 1) } \operatorname{ICC}(\text { dairy, pen, day })=\frac{\sigma_{u}^{2}+\sigma_{v}^{2}+\sigma_{z}^{2}}{\sigma_{u}^{2}+\sigma_{v}^{2}+\sigma_{w}^{2}+\sigma_{z}^{2}+\sigma^{2}} \text {, }
$$

to estimate the similarity in MAP concentrations in environmental samples collected by different collectors 
Table 1. Descriptive statistics for mean cycles-to-threshold values of quantitative real-time PCR for Mycobacterium avium ssp. paratuberculosis on environmental samples tested in duplicate ${ }^{1}$

\begin{tabular}{|c|c|c|c|c|c|c|c|c|c|c|c|c|c|c|}
\hline Dairy & Pens, n & Herd size $^{2}$ & \multicolumn{4}{|c|}{ Day 1} & \multicolumn{4}{|c|}{ Day 3} & \multicolumn{4}{|c|}{ Day 5} \\
\hline 1 & 8 & 1,676 & 32.1 & 2.6 & 32.9 & 3.4 & 33.2 & 1.7 & 32.9 & 2.0 & 33.5 & 1.8 & 32.6 & 1.9 \\
\hline 2 & 11 & 3,577 & 32.8 & 2.6 & 33.2 & 2.8 & 32.9 & 3.1 & 32.5 & 2.8 & 33.1 & 2.6 & 33.2 & 2.4 \\
\hline 3 & 7 & 1,326 & 32.9 & 2.0 & 33.6 & 1.7 & 32.2 & 1.6 & 32.4 & 1.2 & 30.7 & 2.5 & 32.0 & 2.0 \\
\hline
\end{tabular}

${ }^{1}$ Samples were collected from free-stall pens in 4 dairies simultaneously by 2 collectors on 3 occasions while cow movements between pens were minimal.

${ }^{2}$ Herd size is the total number of adult cows represented in environmental sampling of the dairy.

(from the same dairy, from the same pen, and on the same day);

2) ICC (dairy, pen, collector) $=\frac{\sigma_{u}^{2}+\sigma_{v}^{2}+\sigma_{w}^{2}}{\sigma_{u}^{2}+\sigma_{v}^{2}+\sigma_{w}^{2}+\sigma_{z}^{2}+\sigma^{2}}$,

to estimate the similarity in MAP concentrations in environmental samples collected on different days (from the same dairy, from the same pen, and by the same collector); and

$$
\text { 3) } \operatorname{ICC}(\text { dairy, pen })=\frac{\sigma_{u}^{2}+\sigma_{v}^{2}}{\sigma_{u}^{2}+\sigma_{v}^{2}+\sigma_{w}^{2}+\sigma_{z}^{2}+\sigma^{2}} \text {, }
$$

to estimate the similarity in MAP concentrations in environmental samples collected by different collectors on different days (from the same dairy and pen). The 95\% confidence intervals of the ICC were computed using the delta method. The following ranges were used to interpret reliability as estimated by the ICC: $<40 \%$, poor; 41 to $75 \%$, fair to good; $>75 \%$, excellent (Fleiss et al. 2003).

The study results were further analyzed at the replicate and tube level to estimate the variability in MAP concentrations between split environmental samples. Variance components models (equation [1]) were modified to include a random effect for replicate and tube for qrt-PCR and HEYM culture results, respectively. Similarly, the ICC for variability in qrt-PCR and HEYM culture results of split environmental samples given the same dairy, pen, collector, and day of sampling were computed. All analyses were performed using Stata (Stata/IC, version 10.0, StataCorp LP, College Station, TX).

\section{RESULTS}

\section{Descriptive Statistics}

During the study period, a total of $28,77,0$, and 18 cows, including routine hospital cases, were moved between sampled pens on dairies 1, 2, 3 and 4, respectively. Of the 77 cows moved on dairy 2 , a total of 53 were moved from pens with lactating cows to the drycow pen. Tables 1 and 2 present descriptive statistics for qrt-PCR and ln-transformed HEYM culture results, respectively, for MAP in the 180 environmental samples and averaged over pens. The qrt-PCR results ranged from 28 to $40 \mathrm{Ct}$; hence, all were positive for MAP when using the recommended cutoff of the manufacturer of 42 Ct. Sixteen-week HEYM culture results ranged from 0 to $75 \mathrm{cfu} /$ tube, and 21 samples from dairies 1, 2, and 3 had no MAP colony growth on HEYM after $16 \mathrm{wk}$. A total of 44,39,27, and 11 tubes were contaminated and had no detectable growth, out of the 720 tubes cultured from dairies 1, 2, 3, and 4, respectively. Environmental samples from pen 2 in dairy 2 were consistently negative for MAP by HEYM culture; otherwise, at least 1 sample from all the remaining pens had positive MAP cultures. The qrt-PCR results were strongly negatively correlated with both untransformed and ln-transformed HEYM culture results (Spearman's rank correlation, Pearson correlation coefficients $=-0.78, P<0.001$ )

Histograms and normal quantile plots of the $\mathrm{Ct}$ values and $\ln$ mean colony-forming units per tube showed no violation of the normality assumption. The mean of the differences between collectors for Ct values (SD) and in ln mean colony-forming units per tube (SD) were 0.13 (1.44) and -0.13 (1.23), respectively. All but 4 and 8 of the 90 differences between collectors $(30$ pens $\times 3 \mathrm{~d})$ in Ct values and ln mean colony-forming units per tube, respectively, were within the $95 \%$ limits of agreement 
Table 2. Descriptive statistics for natural log of mean Mycobacterium avium ssp. paratuberculosis colony-forming units per tube of environmental samples each cultured on 4 tubes of Herrold's egg yolk medium with mycobactin ${ }^{1}$

\begin{tabular}{|c|c|c|c|c|c|c|c|c|c|c|c|c|c|c|}
\hline \multirow{2}{*}{ Dairy } & \multirow{2}{*}{ Pens, $\mathrm{n}$} & \multirow{2}{*}{ Herd size $^{2}$} & \multicolumn{4}{|c|}{ Day 1} & \multicolumn{4}{|c|}{ Day 3} & \multicolumn{4}{|c|}{ Day 5} \\
\hline & & & \multicolumn{2}{|c|}{ Collector 1} & \multicolumn{2}{|c|}{ Collector 2} & \multicolumn{2}{|c|}{ Collector 1} & \multicolumn{2}{|c|}{ Collector 2} & \multicolumn{2}{|c|}{ Collector 1} & \multicolumn{2}{|c|}{ Collector 2} \\
\hline 1 & 8 & 1,676 & 1.3 & 1.3 & 1.6 & 1.3 & 0.7 & 1.0 & 0.7 & 1.1 & 0.5 & 1.0 & 0.7 & 1.1 \\
\hline 2 & 11 & 3,577 & 1.7 & 1.5 & 1.4 & 1.2 & 1.4 & 1.7 & 1.1 & 1.7 & 1.1 & 1.6 & 1.1 & 1.5 \\
\hline 3 & 7 & 1,326 & 1.9 & 1.1 & 1.2 & 1.3 & 2.1 & 1.4 & 1.7 & 1.0 & 2.5 & 2.1 & 2.2 & 1.7 \\
\hline
\end{tabular}

${ }^{1}$ Samples were collected from free-stall pens in 4 dairies simultaneously by 2 collectors on 3 occasions while cow movements between pens were minimal.

${ }^{2}$ Herd size is the total number of adult cows represented in environmental sampling of the dairy.

between collectors $(-2.69$ to 2.95 and -2.53 to 2.28 for $\mathrm{Ct}$ and ln colony-forming units per tube, respectively). The Bland and Altman plots of the differences in MAP qrt-PCR and HEYM results between collectors against their respective means are shown in Figure 2A and 2B, respectively.

\section{Variance Components Models}

Evaluation of residuals, standardized residuals, and empirical BLUP of both the qrt-PCR and HEYM models indicated good fit. Variability in $\mathrm{Ct}$ values (SE) attributable to dairy, pen, collector, day, and residual error was $<0.01(0.01), 3.73(1.10),<0.01(<0.01), 0.78$ $(0.25)$, and $1.03(0.15)$, respectively. Variability in $\ln$ mean colony-forming units per tube (SE) attributable to dairy, pen, collector, day, and residual error was 0.24 (0.40), $1.22(0.39),<0.01(<0.01), 0.09$ (0.10), and 0.75 $(0.11)$, respectively. Pen and dairy combined accounted for the most variability in MAP concentrations of environmental samples as estimated by qrt-PCR and HEYM culture (67 and $63 \%$, respectively). Collector was a minor source of variability in both outcomes $(<0.01 \%)$. Estimated variability attributable to day was $14 \%$ for qrt-PCR and $3.8 \%$ for HEYM culture results. The ICC and $95 \%$ confidence intervals are presented in Table 3.

Variance components for qrt-PCR and HEYM results at the replicate and tube level indicated that less than 0.1 and $0.1 \%$ of the total variability in MAP concentrations, respectively, was attributable to split-sample testing. The ICC for similarity of results in replicates and tubes given the same collector, sampling day, and pen was excellent $(87 \%)$ and good $(71 \%)$ for qrt-PCR and HEYM results, respectively.

\section{DISCUSSION}

Findings from our study indicate that environmental sampling using a standardized sampling protocol and testing of samples by qrt-PCR and HEYM culture can be used to quantify MAP concentrations reliably in free-stall pens of moderate seroprevalence herds even when implemented by different collectors, at different times, and up to $5 \mathrm{~d}$ after moving cows between pens. The approximately zero mean of differences between collectors for qrt-PCR and HEYM results indicated minimal bias between the study collectors.

The reliability of environmental sampling by different collectors was excellent $(81 \%)$ and good $(67 \%)$ for qrt-PCR and HEYM culture results, respectively, and this justifies comparing quantitative results of environmental samples collected from herds with moderate ELISA prevalence using the standardized protocol by different investigators, such as in Johne's disease control programs or national herd prevalence surveys. The high reliability of environmental sampling by different collectors can be explained by the systematic sampling of the entire surface of each crossover alley in a pen and the collection of fecal slurry already mixed by cow movements through the alleys. In addition, mixing of pen samples using a wooden tongue depressor before dividing them into aliquots for testing may have further contributed to the similarity of samples between collectors.

The reliability of environmental sampling at different times was good (67 and 64\% for qrt-PCR and HEYM culture results, respectively) and was similar to that by different collectors and at different times up to 5 d apart. The reliability of environmental sampling at different times was less than the reliability of sampling by different collectors, and that indicated that variability over time was greater than between collectors. This difference might have been attributable to changes in MAP fecal shedding by cows over the study period and changes in pen population because of cow movement between pens. In addition, samples collected on different days were processed and tested in the order they arrived at the testing laboratory. This was in contrast 


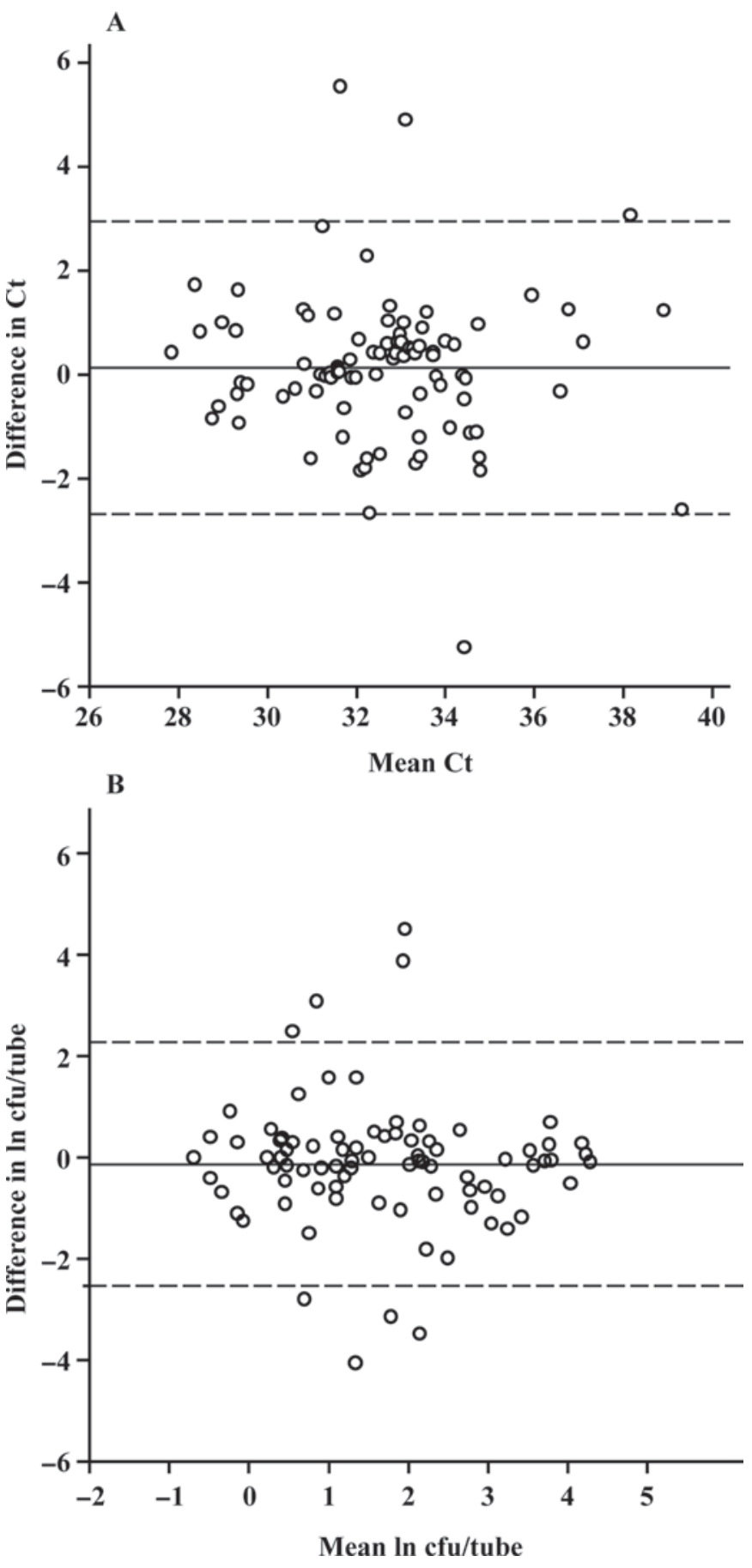

Figure 2. Bland and Altman plots of the differences in results of environmental samples collected simultaneously by 2 collectors and tested for Mycobacterium avium ssp. paratuberculosis by A) quantitative real-time PCR in duplicate and reported as mean cycles-tothreshold (Ct), and B) Herrold's egg yolk medium culture in 4 tubes with mycobactin and reported as natural $\log (\ln )$ transformation of the mean colony-forming units (cfu) per tube. The solid and dashed lines represent the mean of the differences and $95 \%$ limits of agreement between the 2 collectors, respectively. to samples collected by RA and SA on any given day, which were processed and tested in parallel.

The reliability of environmental sampling to quantify MAP was greater for qrt-PCR than HEYM culture, as evident from the higher ICC estimates with qrt-PCR results. In addition, rapid results of qrt-PCR compared with HEYM and the similarity of environmental samples up to $5 \mathrm{~d}$ apart may justify testing environmental samples by qrt-PCR between routine cow pen changes to identify pens with a high MAP bioburden for preferential individual cow testing. Although, the sensitivity and specificity of such a diagnostic strategy to detect high shedders is not known, it may be a rapid and cost-effective diagnostic strategy compared with wholeherd tests or routine lactation-specific testing, such as at dry-off.

The ICC were used to estimate similarity in samples within a group of samples using variance estimates from mixed models. Fixed effects were not considered for dairy, collector, or day because it was not important to test the effect of specific dairies, collectors, or sampling days, but rather to consider the levels of these factors as random selections from their respective groups. Although the choice of an experienced and a recently trained collector was not random, it was considered representative of extreme experience levels in environmental sampling. Furthermore, additional analyses (data not shown) showed no significant effect of collector, day, or number of cows in a pen in models with fixed effects. Although the study herds were a convenience sample of free-stall dairies, they had seroprevalence comparable with other herds in central California, which mostly house cows in free stalls. The study herds had MAP seroprevalence between 3.5 and $4.6 \%$, which was similar to that of 43 randomly selected dairies from the same region, which had an estimated $3.7 \%$ MAP seroprevalence based on 30 randomly selected cows per herd (Adaska and Anderson, 2003).

The extent to which the result of testing an environmental sample for MAP is representative of MAP fecal shedding in cows housed in the pen sampled depends on human, cow, and laboratory-related factors. Humanrelated factors that might affect the representativeness of environmental samples include the uniformity in sample collection, self-preference with respect to locations sampled and time of sampling, experience in sample handling, shipping, and equipment disinfection between pens and herds. The effect of human-related factors may be limited by implementing a standardized sampling protocol, as was done in the present study. Use of new wooden tongue depressors for manually mixing samples from each pen reduced the risk of crosscontamination and was more feasible than the use of 
Table 3. Intraclass correlation coefficients (ICC) for similarity in Mycobacterium avium ssp. paratuberculosis (MAP) concentrations within groups of environmental samples collected from pens in 4 dairies simultaneously by 2 collectors every other day on 3 occasions while cow movements between pens were minimal ${ }^{1}$

\begin{tabular}{|c|c|c|c|c|c|c|c|c|c|}
\hline \multirow[b]{2}{*}{$\mathrm{ICC}$} & \multirow{2}{*}{$\begin{array}{l}\text { Estimate } \\
\text { of similarity } \\
\text { in samples } \\
\text { of different: }\end{array}$} & \multicolumn{4}{|c|}{ qrt-PCR, Ct } & \multicolumn{4}{|c|}{ HEYM, ln cfu/tube } \\
\hline & & Estimate, $\%$ & $\mathrm{SE}$ & Lower & Upper & Estimate, \% & $\mathrm{SE}$ & Lower & Upper \\
\hline ICC (dairy, pen, day) & Collectors & 81.4 & 4.5 & 72.6 & 90.2 & 67.3 & 8.2 & 51.3 & 83.4 \\
\hline ICC (dairy, pen, collector) & Days & 67.3 & 7.3 & 52.9 & 81.6 & 63.5 & 8.5 & 46.8 & 80.2 \\
\hline ICC (dairy, pen) & Collectors, days & 67.3 & 7.3 & 52.9 & 81.6 & 63.5 & 8.5 & 46.8 & 80.2 \\
\hline
\end{tabular}

${ }^{1} \mathrm{MAP}$ concentrations were estimated by mean cycles-to-threshold $(\mathrm{Ct})$ values from quantitative real-time PCR (qrt-PCR) and natural log (ln) of the mean cfu per tube of environmental samples each cultured on 4 tubes of Herrold's egg yolk medium (HEYM) with mycobactin.

${ }^{2} \mathrm{CI}=$ confidence interval.

power tools, which would have been more difficult to clean and disinfect between pens.

Cow-related factors that might affect the variability in MAP concentrations in environmental samples include the number of MAP-infected cows in a pen and the concentration of MAP shed in their feces. Some cows, termed supershedders, may shed at least 10,000 MAP cfu/g of feces (Whitlock et al., 2005). Variability in MAP concentration of environmental samples decreases as the concentration of MAP increases, such as in pens that house supershedders, because of the higher probability of detection of organisms in any single sample. Cow movement between pens may also affect the variability in MAP concentration and may be of importance if environmental samples were to be used to rank pens by MAP bioburden.

Laboratory-related factors include variation in sample processing, testing equipment, and test protocol. Other laboratory-related factors may vary by diagnostic assay, such as DNA sample extraction, as in the case of qrt-PCR. The high similarity of MAP concentrations in replicate environmental samples tested by qrt-PCR or in 4 tubes by HEYM culture suggests an overall low variability attributable to sample processing at the testing laboratory but provides no assessment of analytical variability.

Quantitative results are more useful than binary (positive-negative) results because they provide information about the concentration of MAP in the pen fecal slurry, which might be a useful surveillance tool (Collins et al., 2006). If the only goal of an investigator is to classify a herd as MAP infected based on a single PCR-positive or culture-positive result, then lower reliability might be acceptable, provided multiple locations are tested. In contrast, the reliability of environmental sampling is important when the goal of an investigator is to quantify the MAP bioburden in free-stall pens.

Environmental sampling of gutters in tie stalls has been described previously (Raizman et al., 2004); how- ever, there is no published standardized environmental sampling protocol for the purpose of quantifying the MAP bioburden in tie stalls or dry-lot dairies. Cow movements on hard surfaces in free-stall pens allowed for mixing of individual cow fecal pats and resulted in a homogeneous fecal slurry. In contrast, individual cow fecal pats may not mix as well on large dirt areas, such as in dry-lot pens, or in gutters of tie stalls, and hence may make collection of representative environmental samples for the purpose of quantifying the MAP bioburden in such pens difficult.

For estimating MAP fecal shedding in a cohort of cows, an investigator may collect environmental samples, pooled cow fecal samples, or individual cow fecal samples. Although results of testing pooled cow fecal samples or environmental samples are representative only of their respective cow groups, such aggregate samples offer a reduction in the cost of sample collection and testing by a factor of the number of fecal samples in a pool or the number of cows in a pen, respectively. For estimating the MAP bioburden, such as in dairy 1 housing 1,676 cows with a median pen capacity of 226 cows, environmental sampling of the 8 pens at the dairy was approximately a 210 -fold lower cost than individual sampling and testing of all the cows, or was a 21-fold lower cost than sampling and testing all 168 pools of 10 cow fecal samples.

This study revealed that results of environmental sampling of free-stall dairies housing herds of moderate MAP seroprevalence were comparable between collectors when sampling was performed using a standardized sampling protocol. The study also revealed that qrt-PCR results of environmental samples were comparable when collected up to $5 \mathrm{~d}$ apart and while cow movements between pens were minimal. Hence, qrt-PCR testing of environmental samples may be used as part of a diagnostic strategy to identify pens with a high MAP bioburden between routine cow pen changes on a dairy. 


\section{ACKNOWLEDGMENTS}

This project was supported in part by the USDA-ARS (agreements 58-1265-3-155, 58-1265-3-156, 58-1265-3158, and 58-1265-4-020) for the Regional Dairy Quality Management Alliance, USDA-Cooperative State Research, Education, and Extension Service-National Research Initiative award number 2007-35204, the University of Pennsylvania, School of Veterinary Medicine, Department of Clinical Studies-New Bolton Center, and the Center for Food Animal Health, University of California, Davis.

\section{REFERENCES}

Adaska, J. M., and R. J. Anderson. 2003. Seroprevalence of Johne'sdisease infection in dairy cattle in California, USA. Prev. Vet. Med. 60:255-261.

Berghaus, R. D., T. B. Farver, R. J. Anderson, C. C. Jaravata, and I. A. Gardner. 2006. Environmental sampling for detection of Mycobacterium avium ssp. paratuberculosis on large California dairies. J. Dairy Sci. 89:963-970.

Bland, J. M., and D. G. Altman. 1986. Statistical methods for assessing agreement between two methods of clinical measurement. Lancet 327:307-310.

Collins, M. T., I. A. Gardner, F. B. Garry, A. J. Roussel, and S. J. Wells. 2006. Consensus recommendations on diagnostic testing for the detection of paratuberculosis in cattle in the United States. J. Am. Vet. Med. Assoc. 229:1912-1919.

Crossley, B. M., F. J. Zagmutt-Vergara, T. L. Fyock, R. H. Whitlock, and I. A. Gardner. 2005. Fecal shedding of Mycobacterium avium subsp. paratuberculosis by dairy cows. Vet. Microbiol. 107:257263.

Fleiss, J. L., B. Levin, and M. C. Paik. 2003. The measurement of interrater agreement. Page 604 in Statistical Methods for Rates and Proportions. 3rd ed. Wiley, New York, NY.

Goldstein, H. 1987. Multilevel covariance component models. Biometrika 74:430-431.

Jiang, J. 2007. Linear and Generalized Linear Mixed Models and Their Applications. Springer Series in Statistics. Springer, New York, NY.

Lombard, J. E., B. A. Wagner, R. L. Smith, B. J. McCluskey, B. N. Harris, J. B. Payeur, F. B. Garry, and M. D. Salman. 2006.
Evaluation of environmental sampling and culture to determine Mycobacterium avium subspecies paratuberculosis distribution and herd infection status on US dairy operations. J. Dairy Sci. 89:4163-4171.

Marchenko, Y. 2006. Estimating variance components in Stata. Stata J. $6: 1-21$.

Raizman, E. A., S. J. Wells, S. M. Godden, R. F. Bey, M. J. Oakes, D. C. Bentley, and K. E. Olsen. 2004. The distribution of Mycobacterium avium ssp. paratuberculosis in the environment surrounding Minnesota dairy farms. J. Dairy Sci. 87:2959-2966.

Tavornpanich, S., C. A. Munoz-Zanzi, S. J. Wells, E. A. Raizman, T. E. Carpenter, W. O. Johnson, and I. A. Gardner. 2008. Simulation model for evaluation of testing strategies for detection of paratuberculosis in midwestern US dairy herds. Prev. Vet. Med. 83:65-82.

Tetracore. 2008. VetAlert, Johne's real-time PCR. US Pat. No. 5,985,576. http://www.tetracore.com/pdfs/Johnes-032508.pdf Assignee: Tracy Fecteau.

USDA. 2002. NAHMS Johne's disease demonstration herd project. http://nahms.aphis.usda.gov/jddh/index.htm Accessed Apr. 27, 2009.

USDA. 2005. Johne's disease on U.S. dairy operations, 2002. No. N427.0205. USDA-Animal and Plant Health Inspection ServiceVeterinary Services, Centers for Epidemiology and Animal Health, National Animal Health Monitoring System, Fort Collins, CO. http://www.aphis.usda.gov/vs/ceah/ncahs/nahms/dairy/ dairy02/Dairy02_Johnes_rept.pdf Accessed Apr. 27, 2009.

USDA. 2006. Uniform program standards for the voluntary bovine Johne's disease control program. APHIS 91-45-016. USDAAnimal and Plant Health Inspection Service-Veterinary Services, Washington, DC. http://www.aphis.usda.gov/animal_health/ animal_diseases/johnes/downloads/johnes-umr.pdf Accessed Apr. 27,2009 .

USDA. 2008a. Johne's disease on US dairies, 1991-2007. No. N521.0408. http://nahms.aphis.usda.gov/dairy/dairy07/Dairy2007_Johnes. pdf Accessed Apr. 27, 2009.

USDA. 2008b. USDA-approved laboratories - Johne's disease organismbased methods. http://www.aphis.usda.gov/animal_health/lab_ info_services/downloads/ApprovedLabs_Johnes_organism.pdf Accessed Apr. 27, 2009.

Whitlock, R. H., R. W. Sweeney, and T. Fyock. 2005. MAP supershedders: Another factor in the control of Johne's disease. Pages 193-194 in Proc. 38th Am. Assoc. Bov. Pract. Conf., Salt Lake City, UT. Am. Assoc. Bovine Pract., Frontier Printers Inc., Stillwater, OK 